\title{
MADRES DE LA NOVELA HISTÓRICA ITALIANA
}

\section{María Reyes Ferrer}

mariarefe@gmail.com

Universidad de Murcia

Recibido: 28-02-2015

Aceptado: 27-04-2015

\section{Resumen}

En este artículo se analiza el papel que jugaron las escritoras italianas en la configuración de uno de los géneros literarios que mayor repercusión alcanzó en Italia: la novela histórica. Desde sus inicios, este género se ha relacionado casi de manera exclusiva con Alessandro Manzoni, más conocido como el padre de la novela histórica italiana. No obstante, las mujeres también participaron del género histórico y, según varios estudios, se desviaron ligeramente del canon tradicional para buscar nuevas formas y explorar una temática que enriquecerá el género y aportará una nueva visión de la historia y de la literatura.

Palabras clave: Escritoras, novela histórica, Italia, literatura, historia, Alessandro Manzoni, tradición literaria.

\begin{abstract}
This article analyzes the role that Italian women writers played in the configuration of one of the literary genres, which had a greater impact in Italy: the historical novel. Ever since its origins, this genre has been related almost exclusively with Alessandro Manzoni, best known as the father of Italian historical novel. However, women also played a fundamental role in the historical genre, and according to different studies, they slightly diverted from the traditional canon in order to try new methods and explore new themes, which would ultimately enrich the genre and provide with an innovative view of history and literature.
\end{abstract}

Key words: Female writers, historic novel, Italy, literature, history, Alessandro Manzoni, literary tradition 


\section{Introducción}

En la mayor parte de los países europeos, la novela histórica alcanzó su plenitud durante el romanticismo especialmente gracias al novedoso trabajo de Walter Scott, considerado el primer impulsor de la novela histórica, o Alessandro Manzoni que, influido por $\mathrm{Scott}^{1}$ en lo referente a la novedad del género, será recordado como el padre del romanzo storico en Italia y su obra será un referente para dicho género. Dado que la historiografía constituye el principal punto de apoyo de la novela histórica, el nacimiento de dicho género literario pone de manifiesto la importancia que adquiere la disciplina histórica. Es más, se podría afirmar que el origen del género es fruto de un creciente interés por los acontecimientos de un tiempo pasado y la consiguiente rememoración de hechos ya vividos en otras épocas. Existe un deseo de hacer historia que, además, pone de manifiesto la cuestión romántica literaria sobre la posible relación que existe entre la historia y la invención. Esta particular inclinación por descubrir la historia y, a su vez, vincularla a la literatura, no nace de forma casual sino que, claramente, se manifiesta en un período caracterizado por la rapidez de los acontecimientos en un momento en el que las viejas visiones del mundo sucumben frente al avance de otras nuevas, como argumentará Northrop Frye (1977) al hacer referencia a las obras de Walter Scott.

A lo largo del siglo XX, la novela histórica seguirá creciendo y desarrollándose, sufrirá una metamorfosis -y no una crisis ${ }^{2}$ - debido en parte al duro revés que experimenta uno de sus pilares básicos, la historiografía tradicional. En este siglo, motivado por distintos factores, se deconstruyen algunos de los indicadores históricos que anteriormente se consideraban definitivos y absolutos en su relación con la historia, como la objetividad o la totalidad de los hechos. De esta manera, la disciplina histórica se aleja de su condición de discurso absolutista,

\footnotetext{
${ }^{1}$ El éxito de Scott en Italia se debe en buena parte a la situación que atravesaba el país en los inicios del Ottocento. Italia estaba deseosa de tener una identidad propia, alejada de la sociedad del ancien régime, y Scott, como proponía Roberto Bigazzi (2007), supo captar la necesidad de identidad de los países: "Scott aveva offerto lo strumento per rileggere la storia (e magari cambiarne i protagonisti) alla luce dei valori appunto borghesi, liberali, post-rivoluzionari, cioè quell'insieme di principi, sentimienti, comportamenti, in rapporto alla vita e alla società, che confluiscono nel concetto di 'costumi' (la grande parola d'ordine tra Sette e Ottocento)" (Bigazzi, 2007: 28).

${ }^{2}$ Margherita Ganeri en su obra Il romanzo storico in Italia (1999) sugiere que el género histórico nunca estuvo en crisis sino que, más bien, sufrió una redefinición, adaptándose a los tiempos y a los nuevos modelos: "Fratture, rotture e svolte cruciali non rendono però impraticabile, pur nella discontinuità, il riconoscimento dei tratti di continuità" (Ibídem: 49).
} 
único y verdadero, algo que ya adelantaban reconocidos críticos de la postmodernidad como Linda Hutcheon, Frederic Jameson o Hyden White. Esta nueva concepción histórica permite echar la vista atrás y ver los espacios en blanco que la Historia deja, siendo posible pensar en el concepto de Historia desde una posición más marginal y subjetiva, con la posibilidad de hacer “una historia desde abajo". Si se extrapola este pensamiento al campo literario, concretamente al análisis de la producción novelística, "parece existir un nuevo deseo de pensar históricamente, y pensar históricamente a día de hoy significa pensar críticamente y contextualmente" (Hutcheon, 1988: 88).

Las novelas en general, y las escritas por mujeres en particular, se llenan de personajes marginales que aceptan de forma un tanto problemática las estructuras sociales y existenciales, reflejando a través de ellos las reticencias que los escritores y escritoras tienen a la hora de asumir su situación en el mundo y el poder establecido (Del Pardo, 1999). A este respecto, el uso de la historia en la escritura literaria femenina aparece con un fuerte componente crítico hacia la posición de la mujer, pues esta se niega a aceptar ese "poder establecido" y el lugar marginal, escasamente representado, que ocupa en el mundo. De hecho, el resultado de la incorporación de la mujer a la narrativa histórica supone un evidente distanciamiento, en ciertos aspectos, de la novela histórica tradicional ya que, como sugiere Navarro Salazar (2006: 194), esta "aparece enriquecida con formas específicas que, bien sea en sus intenciones, bien sea en la elección de los personajes y ambientes, apuntan a delimitar y crear una nueva historia".

Llegados a este punto, estimo necesario hacer dos consideraciones: en primer lugar, la novela histórica italiana ha ido siempre acompañada de la autoría de Alessandro Manzoni, conocido como el "padre" de la novela histórica en Italia, quien estableció un canon literario que servirá de modelo para tantos otros escritores que practiquen el género histórico. Sin embargo, como se ha comentado, las aportaciones que hacen las mujeres a la novela histórica han alcanzado gran relevancia e incluso una cierta especificidad propia de la escritura femenina. Por tanto, si se toma como punto de referencia la afirmación de Navarro Salazar y la existencia de una novela histórica femenina, cabría preguntarse si en los orígenes del género en Italia existieron "madres" de la novela histórica, o por el contrario son todas las escritoras "hijas" de una tradición manzoniana de la que más tarde parecen desvincularse para cultivar formas propias. A continuación, se trazará una línea de investigación que se centrará en las relaciones de las mujeres y la disciplina histórica y literaria para, en un segundo momento, 
analizar qué escritoras practicaron el género de la novela histórica desde su llegada a Italia para estudiar si existieron deviaciones respecto a la novela histórica tradicional y, por tanto, es posible hablar de esas "madres" literarias.

\section{Relaciones entre mujer, historia y literatura: hacia una nueva concepción de las disciplinas}

En primer lugar, es oportuno remarcar que con la acuñación de "madres" de la novela histórica no se pretende marcar una diferencia biológica-literaria o trazar dos historias de la literatura diversas, sino más bien reconocer y evidenciar una realidad: las mujeres escriben y escribieron en un tiempo pasado, moviéndose por los diversos géneros literarios y demostrando su valía como literatas. Chiara Stinghi (2001) hace una acertada diferencia entre las escritoras: las anteriores a la mitad del Settecento, es decir, las que escribían para sí mismas de manera privada y a escondidas, y las escritoras de mediados y finales del Ottocento hasta ahora, quienes salieron a la luz y lograron imponerse al mercado editorial. Que las mujeres practicaran la escritura significa que, cualquier intento de anulación u omisión de estas, como viene sucediendo desde el pasado, contribuye a un empobrecimiento de la literatura ${ }^{3}$, y de la cultura en general, como sugiere Laura Fortini: “[...] la loro presenza costituisce elemento di arricchimento e di interrogazione feconda per la letteratura italiana, non tanto per un criterio meramente aggiuntivo, quanto invece per la qualità del loro contributo alla letteratura italiana" (2010: 178). El hecho de que se evite la inclusión de las escritoras en una tradición literaria se

\footnotetext{
${ }^{3}$ Antes de pasar a una lectura más detallada sobre las escritoras, es oportuno puntualizar que en los estudios de literatura escrita por mujeres, a pesar de los grandes avances y esfuerzos realizados -y todavía hoy- en el campo, todavía existe un largo camino por recorrer. En primer lugar, en términos biográficos, se ha perdido mucha información sobre mujeres que a lo largo del tiempo pasaron desapercibidas e/o infravaloradas por el hecho de ser mujer y escribir, un domino exclusivo masculino. En segundo lugar, la crítica ha prestado mucha menos atención a las obras escritas por mujeres $\mathrm{y}$, a día de hoy, realizar un análisis completo de las mujeres que practicaron la novela histórica en el Ottocento conllevaría un estudio aparte. Para tal estudio, al igual que para el presente, y en el campo de la literatura femenina en general, considero de gran importancia -y de gran ayuda, especialmente- las obras de Costa-Zalesswo, N., Scrittrici italiane dal XIII al XX secolo: testi e critica, 1982; Santoro, A., Narratrici italiane dell'Ottocento, 1987; Wood, S., Italian women's writing 1860-1994, 1995; Trigila, M., Letteratura al femminile. Dalle origini ai nostri giorni in Italia, 2004; Ciopponi, M., Parola di donne. Otto secoli di letteratura italiana al femminile, 2006, entre tantos otros.
} 
debe, principalmente, a que estas han sido consideradas por la crítica como "casos aislados", siguiendo la afirmación de Mercedes Arriaga (2008) y, por lo tanto, de escaso interés para la disciplina. La crítica literaria feminista ha puesto especial énfasis en la reconstrucción de una tradición literaria en femenino, donde las escritoras adquieran una visibilidad y un reconocimiento a su trabajo, más allá de los juicios de valor condicionados por el género. Para ello, Arriaga señala la importancia de tener en cuenta el concepto de genealogía femenina ya que "incide en conceptos literarios como la influencia, modelo, traducción, imitación, continuidad, tradición de escritura y utilización de estrategias retóricas parecidos entre escritoras de periodos diferentes" (2008: 56). La teoría del "orden simbólico de la madre", elaborada por Luisa Muraro (1991) y a propósito de las herencias culturales, persigue la construcción de una genealogía femenina cultural y denuncia el orden simbólico del padre que había impuesto unos parámetros ideológicos y creativos con un claro molde masculino.

"Soy consciente de lo problemático que es designar como masculina una cultura a la cual también han contribuido, más o menos activamente, también las mujeres, comenzando por mi modesta aportación. La llamo masculina basándome en el criterio de la autoridad que prevalece en el acto, fundamental para toda cultura, de la mediación. Es masculina la cultura en la cual la autoridad se identifica con preferencia en el ser hombre. Se trata de un criterio simbólico (la autoridad es simbólica, o no es tal). La cultura en la que me expreso en este momento presente es femenina; también forman parte de ella algunos hombres, como Platón o mi padre o mi maestro de metafísica, pero es cultura femenina porque lo es la autoridad mediadora para mí" (Muraro, 1994: 132).

Muraro invita a reflexionar acerca de la invisibilidad o la escasa presencia femenina en la historia y en la literatura a través de la "ley Lina Vannucci", un término acuñado por ella misma y que utiliza para hacer hincapié en la desaparición de la mujer de los códigos culturales y la necesidad de trabajar, como propone María Milagros Rivera a partir de la teoría de Muraro, en esos códigos culturales para "captar cuales son o pueden ser significativos de esa presencia femenina que la experiencia indica y que apenas se ve en las enciclopedias" (1996: 59).

Respecto a la relación de la mujer con la disciplina histórica, si bien pocas veces es reconocida la mujer como escritora, todavía más difícil resulta encontrar mujeres que se 
dedicaran al estudio e investigación histórica en sus diversas formas, pues dicha disciplina parece ser un campo reservado al género masculino:

\begin{abstract}
"Terreno molto più tardivamente esplorato in tal senso rispetto ad altre forme del sapere e della scrittura, la storiografia e, più in generale, la conoscenza e la ricostruzione del passato, sono state prevalentemente configurate, almeno fino al primo dopoguerra, quale ambito "senza donne": nel quale cioè, ancora in piena età contemporanea, la presenza femminile risultava debole, eccezionale, del tutto marginale" (Casalena, 2003: IX).
\end{abstract}

No obstante, y gracias en parte al estudio de Maria Pia Casalena, es posible analizar cómo las mujeres despertaron un gran interés por la historia y, además, colaboraron aportando nuevos datos, nuevas visiones, aunque con escasa repercusión y difusión de los resultados. A medida que se iba constituyendo la identidad nacional en la época del Risorgimento italiano, la presencia y el asentamiento de una disciplina histórica practicada por mujeres aumentaba:

\begin{abstract}
"La forte domanda di medaglioni e memorie, l'espansione del mercato editoriale legato a questo tipo di produzione, l'esigenza di ammettere le donne -come autrici e come lettrici- in questo vasto movimento intellettuale senza pregiudicare la stabilità dei ruoli di genere, permisero alle donne italiane di diventare sempre più numerose scrittrici e lettrici di storia nazionale già prima dell’Unità" (Ibídem: XXXII).
\end{abstract}

Es más, basándose en los datos recogidos, Casalena no duda en afirmar que el Risorgimento representa "la chiave d'accesso autentica e l'ambito privilegiato di esercizio e diffusione della scrittura storica femminile" (Ibídem). A través de su estudio se diferencian tres períodos históricos claves: 1800-1861, años en los que es posible observar el nacimiento de un pensamiento libre y democrático de Italia que marca el camino hacia la Unidad; 1861-1922, se definen como unos años de asentamiento y afirmación hasta el fascismo; 1923-1945, la historia está a merced del régimen fascista. Analizando la evolución de las aportaciones en la historia en cada una de las fases es posible advertir las trasformaciones del rol femenino en el ámbito de la investigación histórica: "le ragioni della storia politica si intrecciano infatti inscindibilmente con quelle del maggiore o minore coinvolgimento delle donne nella costruzione dell'identità nazionale" (Gentile, n.d). Sin embargo, mientras que la aportación de 
las mujeres a los estudios de la historia resulta evidente ${ }^{4}$, menos esclarecedora parece la relación que tuvieron las escritoras con la novela histórica.

\section{Madres de la novela histórica italiana}

No es tarea fácil -ni estrictamente necesaria, añadiría- delimitar un principio y un final preciso en la vida de la novela histórica pero, para comenzar a trazar el origen de la práctica femenina en materia histórico-literaria, considero de gran importancia comenzar por Diodata Saluzzo Roero y su obra Il castello di Binasco, novela ambientada en el Trecento y publicada en el 1819. Dicha novela viene definida como el "embrione del romanzo storico" (Casarini, 2010) que anticipó la publicación de I Promessi Sposi. Resulta curioso analizar la amplia correspondencia mantenida entre Saluzzo Roero y Manzoni, este último generalmente poco dispuesto a entrar en debates literarios excepto con ella, a quien hace ciertas consultas ${ }^{6}$. En estas cartas, al igual que en otras que el propio Manzoni escribió, este se declara ajeno a los debates literarios sobre el romanzo storico, algo en lo que Saluzzo Roero estaba muy implicada y participaba de forma muy activa. Cuando se habla de su novela, - aunque generalmente se estudia más su faceta poética-, se suele describir como una narración con unos aspectos históricos todavía por pulir, no muy bien definidos, o como "un breve racconto storico" (Bazzoni, 1906: 194), una novella e incluso un romanzo. En su ensayo crítico, Bazzoni comenta la obra de Diodata Saluzzo, aportando lo siguiente:

\footnotetext{
${ }^{4}$ Ver Casalena, M.P., Scritti storici di donne italiane. Bibliografia 1800-1945, 2003. En el estudio recoge las diversas obras y escritos de carácter histórico escritas por mujeres durante el Ottocento y parte del Novecento. Los datos aportados por Casalena no dejan lugar a duda de que las mujeres fueron muy activas en sus aportaciones, mostraron -y siguen mostrando-un gran interés y eficiencia en su estudio de la disciplina histórica, por lo que los motivos de su "silencio" no se reducen a otros factores que la hegemonía del género masculino sobre los diversos aspectos intelectuales.

${ }^{5}$ Cfr. Prosio, P.M., "Agli albori del romanzo storico in Piemonte: Le 'Novelle' di Diodata Saluzzo" en Ludovico di Breme e il programma dei romantici italiani. Atti del Convegno di studio, Torino, 21-22 de octubre 1983, Torino, 1984, pp.169-182.

6 "Si dà quasi intera la lettera di Manzoni a Diodata Saluzzo di Roero del 16 novembre 1827 perché costituisce il modello principe della corrispondenza che possiamo chiamare di consulenza letteraria. È nota la ripugnanza di Manzoni alla dispute letterarie, salvo che non si trattasse di giustificare un proprio lavoro" (Nencioni, 1993: 194)
} 
“[...] attenne al genere più facile della novella storica, più o meno breve, ma non mai complessa e pretenziosa come il romanzo. [... ] Chi trattò primo in Italia questo genere fu, che io sappia, la contessa Diodata Saluzzo Roero, colei che la Biblioteca Italiana chiamava la Pantasilea dei romantici, col breve racconto storico Il Castello di Binasco, comparso nel 1819, sul Ricoglitore; poi non m'è riuscito di ritrovare altri cultori della novella storica in prosa fino al 1827 [...]" (Ibídem).

¿Es una novella, un racconto o un romanzo? Tras leer diversos comentarios ${ }^{7}$ a la obra, se llega a la conclusión que su obra es más que una novella y se acerca mucho a lo que se conocerá como romanzo storico. Prueba de ello es que muchos escritores tendrán buena cuenta del trabajo de Diodata Saluzzo Roero a la hora de componer sus narrativas históricas.

No sólo es importante tener en cuenta que su obra supuso una fuente de inspiración - o un posible modelo de imitación para muchos escritores, entre ellos Manzoni quien, en varias ocasiones, reconoce el talento y la admiración que siente por ella- sino que además introduce un tema que será común en la escritura femenina, histórica o no, que es la condición de las mujeres. Es precisamente en la novela Il Castello di Bignasco que Diodata Saluzzo introduce en el género histórico la violenta muerte de Beatrice di Tenda. Además, el hecho de reflejar en la novela las miserables condiciones que rodean a las mujeres aparecerá como una constante en la trama de las ficciones escritas por mujeres, desvelando diversas realidades a través de la narrativa.

Siguiendo esta línea, otra escritora que no se debe pasar por alto es Enrichetta Caracciolo Forino y su obra I misteri del chiostro napoletano (1864). A pesar de que dicha obra no se podría considerar novela histórica como tal porque más bien pertenece al género de la memorialística, sí que introduce elementos históricos y además hace una crítica feroz, a través de su experiencia personal, sobre la condición de represión de la mujer y la situación cultural e histórica del Mezzogiorno. Tal y como Trigila la define, la obra presenta influencias de varios modelos literarios: "La struttura della prima parte obbedisce al romanzo settecentesco d'avventura e al romanzo di denuncia di stampo illuminista, mentre nella seconda parte

\footnotetext{
${ }^{7}$ Algunos de los estudios a su obra son: Verga, E., "Beatrice" en Enciclopedia Italiana Treccani, 1930; Bertolotti, D., Il Nuovo Raccogliotore Ossia Archivi d'ogni letteratura antica e moderna, 1830; Ferrario, V., "Novelle di Diodata Saluzzo Roero" en L'Eco, giornale di scienze, lettere, arti, mode e teatri, , vol.3, 1830; Brighenti, M., Letteratura e cultura nei periodici veronesi di fine Ottocento, 2011.
} 
s'intrecciano motivi del romanzo storico-risorgimentale e del romanzo d'appendice" (Trigila, 2004:110).

Maria Rosa Cutrufelli, en las notas críticas a la obra, declara que la novela supone "algo más" que las memorias de Caracciolo; se puede considerar como una historia común, y sumergida, de tantas mujeres a las que les da voz en primera persona: "Eppure si sente in alcune affermazioni, nel modo stesso di affrontare taluni argomenti, l'eco, se non la consapevolezza, di una storia, già lunga anche in Italia, di lotte emancipazioniste e di rivendicazioni femminili" (Cutrufelli, 1998: 7). Al igual que Caracciolo, la escritora Luigia Codemo se desenvuelve a la perfección en los diversos géneros de novela, ensayo, novelle y poesía. En términos generales, su obra es muy vasta y se caracteriza por una escritura poco homogénea pero con un punto común: un mundo femenino lleno de mujeres humildes y las escenas domésticas.

"La narrativa di Luigia Codemo ha una filiazione diretta dal romanzo storico manzoniano, da cui mutua l'interesse per le "genti meccaniche e di piccolo affare", ma attinge anche al vasto repertorio delle forme brevi, degli exempla, dei racconti morali, delle "storie vere" che sembrano riprese dalle colonne della 'Gazzetta Veneta' di Gozzi, delle goldoniane chiacchiere da campiello, senza trascurare le storie sentimentali e romantiche dei racconti d'appendice" (Chemello, 2012: $1)$.

Los argumentos son de índole patriótica y social con ciertos aires manzonianos, como sostiene Chemello, en su elección de los humildes y la profundidad psicológica de los personajes. No obstante, al igual que Caracciolo, esta escritora se acerca al género de novela histórica en algunos aspectos, como los ya citados, aunque es cierto que muestra una cierta preferencia por un estilo menos rígido que le permite divagar entre los diversos módulos narrativos. Las historias de sus obras son más bien "escenas", algo ya deducible por el título de sus obras como: Le memorie d'un contadino: scene domestiche (1856), Berta: prima cronaca d'un anonimo: scene domestiche (1858), La rivoluzione in casa: scene della vita italiana (1869), Miserie e splendori della povera gente: scene popolari (1876), entre otras. En ellas la escritora alterna las diversas modalidades de escritura y, como Chemello apunta, "sullo sfondo, qualche cenno discreto, qualche allusione pudica alla storia delle guerre risorgimentali, alla tensione ideale che aveva sorretto la generazione protagonista della rivoluzione del 1848" 
(Chemello, 2012: 1). Se podría decir que su obra se mueve entre la escuela manzoniana que apoya la novela histórica y propugna una literatura pedagógica y moral, y una escuela realista que progresivamente traslada la visión de la historia a la contemporaneidad.

Posiblemente, dos de las escritoras que han gozado de mayor fortuna crítica han sido Cristina Trivulzio di Belgioioso y Carolina Invernizio. Sin embargo, y al igual que en el caso de las escritoras arriba mencionadas, resulta prácticamente imposible encontrar un testimonio de crítica literaria donde aparezcan estas mujeres como escritoras que guardan algún tipo de relación con el género histórico. Todas ellas, como se ha comentado de las anteriores, parecen desviarse de una norma impuesta sobre la novela histórica y, por tanto, se desvinculan ligeramente de un canon establecido principalmente por los escritores ${ }^{8}$. De hecho, resulta muy interesante analizar cómo las obras de estas mujeres suelen definirse como eclécticas y heterogéneas por moverse en varios estilos. De ser así, puede considerarse un tanto paradójico encontrar nombres masculinos asociados de forma categórica a un género literario mientras que las escritoras parecen divagar entre los géneros sin encontrar un lugar, su lugar.

Por tanto, es posible afirmar que las escritoras, en numerosas ocasiones, quedan fuera de los parámetros literarios ya que estos se elaboraron sin tener en cuenta una escritura femenina ${ }^{9}$. Es por ello que, tal y como se mencionó anteriormente acerca de la relación de las mujeres y la novela histórica en el Ottocento, muchas de las producciones femeninas pueden ser definidas como eclécticas porque, en primer lugar, la literatura no ha ni contemplado ni integrado la participación de las escritoras y, en consecuencia, las obras no satisfacen ningún patrón literario impuesto por los escritores y su hegemonía en el ámbito literario. En segundo lugar, y algo que ya avanzaba Casalena (2003: IX) en el terreno de la escritura de la historia, es necesario tener en cuenta que las mujeres no siempre han tenido acceso a las fuentes históricas, "agli archivi, a tutti gli strumenti necessari alla ricostruzione e interpretazione del passato" y, por tanto, dicha falta de medios, entre otros motivos, contribuye a suplir las carencias con otros

\footnotetext{
8 Tanto en la literatura como en la historia, existen unas normas y cánones establecidos por un pensamiento y una producción puramente masculina. Respecto a la historia, uno de los primeros trabajos en que refleja la influencia del género en dicha disciplina es la obra de B.G. Smith, The Gender of History: Men, Women, and the Historical Practice, 1996. La autora ya apuntaba que la historia "objetiva" parecía ser sólo contada por hombres, y las aportaciones femeninas eran sistemáticamente descartadas por estos, considerando los textos científicos escritos por mujeres superficiales, repetitivos e "indicativos de vanidad o amor al lujo- connotando lo sexual en lugar de lo racional" (Smith, 1996: 3).

${ }^{9}$ Cfr. Bo, C., "Pesentazione", AAVV, Scrittrici d'Italia, Genova, Costa Nolan, 1995.
} 
estilos y géneros de escritura ${ }^{10}$. En tercer lugar, son las propias escritoras las que no sintiéndose 'cómodas'- por una más que posible y comprensible falta de identificación- en los géneros literarios prevalentemente masculinos, deciden probar y desafiar con nuevas formas y nuevas perspectivas.

"Come donne di cultura, insoddisfatte del sapere offerto loro dalla scuola, queste figure cercano di colmare questi vuoti attraverso la lettura: una lettura individuale e casalinga, spesso furtiva e colpevole. Appassionate lettrici si immergono in romanzi, novelle, riviste e in queste letture disordinate vi proiettano emozioni invano cercate e la loro fantasia vi trova appagamento" (Stinghi, 2001).

Sin ir más lejos Karoline Rörig (2010: 36) al hablar de Cristina Trivulzio, la define como "una pensatrice eclettica e poco sistematica". La pasión que la princesa di Belgioioso sintió por la historiografía y la contribución que hizo a esta disciplina son incuestionables y, como la estudiosa afirma, "[...] si appassionò e accettò la storiografia come una sfida intellettuale, dimostrando grandi ambizioni [...] apprese i princìpi base del lavoro scientifico: la lettura sistematica, il rapporto critico con le fonti, la selezione delle citazioni e l'uso preciso dei termini” (Ibídem: 35).

No obstante, son escasas las veces que se ha tenido en cuenta su visión de la historia y su aportación a la historiografía, quedando fuera de los debates de la época debido a su condición de mujer ${ }^{11}$. Poco a poco, su nombre ha ido retomando la fuerza que tuvo en otro tiempo a medida que los estudios de género han avanzado, lo que ha permitido rescatar muchos de sus escritos del pasado, y del olvido. Los aspectos más comentados a día de hoy sean quizás

\footnotetext{
${ }^{10}$ Casalena apunta al período de tiempo que va entre 1910 y 1940, como los años de formación y afirmación de un reducido "gruppo di storiche" académicas y profesionales de la historia. Aunque, como se ha dicho anteriormente y citando a Casalena, existió un gran movimiento intelectual que hacía de las mujeres escritoras y lectoras de historia, teniendo cierta participación dentro de la disciplina, no llegarán a tener ni la mitad de privilegios, tanto de formación como de aceptación en los distintos sectores sociales, culturales e intelectuales, que sus coetáneos masculinos.

${ }^{11}$ De entre las aportaciones a la historia de Belgiojoso cito algunas como Essai sur la formation du dogma catholique (1842); Studi intorno alla storia della Lombardia degli ultimi trent'anni e delle cagioni del difetto d'energia dei lombardi. Manoscritto in francese di un lombardo voltato italiano da un francese (1847); L'Italie et la révolution italienne de 1848. I: L'insurrection lombarde et le gouvernement provisoire de Milan. II: la guerre de Lombardie det la capitulation de Milan. III: La révolution et la République de Venise. IV: L'insurrection du Tyrol en "Revue des deux mondes" (1848) entre otras publicaciones. Para ampliar más información sobre sus diversas facetas de estudio, ver Arriaga Flórez, M., "Cristina Trivulzio Belgioioso en la prensa", en Bernard. M., Papel de Mujeres. Mujeres de papel, Bergamo, Bergamo University Press, 2008. p.10.
} 
su pensamiento político de género y su faceta periodística; en lo que respecta a su obra literaria, esta parece tener un menor eco entre la crítica, aunque no por ello es menos interesante el punto de vista que adoptan en su narrativa. Es más, sería un error desvincular su pensamiento de género de sus novelas, ya que las mujeres y, especialmente, la condición de las mujeres, son el centro de su obra tanto literaria como política. Cristina Trivulzio, por ejemplo, sorprende con obras como Scènes del la Turque (1858) en la que recoge los relatos de Un principe Curdo, Emina y Le due mogli di Ismail Bey, siendo una de las primeras mujeres que afrontó el tema del harem, contribuyendo así a la difusión y el conocimiento de la situación de la mujer en los países orientales.

Al tratar la figura de Carolina Invernizio y su obra, esta generalmente viene vinculada al fenómeno sociológico ${ }^{12}$ desatado por su literatura, algo que ha pasado muy de puntillas por la crítica literaria. Como Arslan mantiene, esta escritora, al igual que la Marchesa Colombi, Annie Vivanti o tantas otras, se ven envueltas en una tradición femenina literaria donde existen novelas leídas por mujeres, escritas por mujeres y relacionadas con temas típicamente asociados al género femenino, encontrando en esta literatura un espacio "che le è negato nella letteratura 'vera', ma anche la possibilità di sicuri guadagni” (Arslan, 1998: 16). Por tanto, no sólo se comienza a construir un espacio propio sino también la posibilidad, al igual que Trivulzio, de dar un salto al mercado editorial y, por tanto, obtener una cierta independencia económica. Teniendo en cuenta estos motivos, Arslan apunta que el verdadero valor de esta literatura es más bien de naturaleza sociológica, ya que el público viene muy bien definido y, además, se revela la existencia de un grupo de escritoras que producen las necesidades de este público, influyendo en el comportamiento y el deleite de este.

Las escritoras de finales del Ottocento merecen una atención, pero lo de Carolina Invernizio, el "fenómeno Invernizio" es un estudio aparte. Su producción logra abrirse camino en el campo masculino del "romanzo d'appendice", el folletín. De entre sus novelas, alrededor de 130, muchas de ellas no dejan de tener una mirada al pasado, como el caso de $I l$ bacio di una morta (1886), La trovatella di Milano (1889) o Il segreto di un bandito (1898) entre otros. Estas son de sus primeras novelas, cuando todavía tentaba lo que sería su gran éxito, el

\footnotetext{
${ }^{12}$ Varios títulos de sus novelas llevan la coletilla de "romanzo storico sociale", tal y como su editor, Adriano Salani, define sus novelas.
} 
feuilleton ${ }^{13}$. Tanto en la primera como en la segunda novela se recrea un escenario histórico de gran importancia: "le cinque giornate", uno de los episodios más conocidos del Risorgimento italiano. En La trovatella se rememora dicho período de la mano de personajes ficticios, como Annetta Durini, o personajes históricos reales, como Luisa Battistotti Sassi, una de las muchas heroínas de la época que recrea. En la tercera novela citada, Carolina Invernizio trata con la polémica figura de Filippo Curletti, perteneciente al cuerpo de la policía y más tarde acusado por formar parte de la banda de "la Cocca". De entre sus novelas, históricas o no y al igual que en las otras escritoras, destaca un marcado interés por los personajes femeninos:

\begin{abstract}
"Las protagonistas de sus novelas son principalmente mujeres que afrontan problemas amorosos y familiares. Por vez primera en la literatura italiana las mujeres asumen un papel ejecutor y plenamente activo. Se nos muestran como salvadoras de sus maridos, hijos o amantes. Estas sustituyen al superhombre descrito en las novelas de Dumas y Sue. Esta ferviente voluntad emancipadora de la autora convierte al hombre en mera comparsa, relegándolo a un rol secundario" (Romano, 2011: 203).
\end{abstract}

Otra vez más, la figura de la mujer ocupa el centro de la invención literaria de las escritoras para la representación de una realidad histórica y/o social. Y no es la única característica que comparten las escritoras de este período sino que, a modo de ejemplo, Invernizio también será criticada no tanto por un eclecticismo literario sino más bien por la variedad de temas y sensaciones que sus novelas pueden suscitar: "potrà gratificarsi il cacciatore di kitsch, sdegnarsi la femminista, allenarsi il narratologo, riflettere il sociologo, divertirsi il non addetto ai lavori" (Federzoni, 1979: 33).

\footnotetext{
${ }^{13}$ Como Federzoni afirma, "un paragone con il romanzo realista dell'800 si impone sia per la sua rilevanza storica che per la pertinenza metodologica: romanzo storico prima, romanzo realistico poi, sono i generi con cui si è confrontata la letteratura d'appendice, raccogliendone gli influssi e le suggestioni estetiche, per poi servirle omogeneizzate al suo pubblico popolare" (1979: 54). Tanto por ciertas declaraciones como por su escritura, se sabe que Carolina Invernizio tenía un amplio conocimiento de la novela histórica italiana de la época.
} 


\section{Conclusiones}

Una vez comentadas brevemente las obras de estas escritoras es oportuno hacer dos consideraciones en consonancia con las dos cuestiones planteadas al inicio de este estudio. En primer lugar, y respecto a la primera cuestión, es posible afirmar que existieron 'madres' en el género literario histórico y, es más, es difícil encontrar herederas, 'hijas' de la tradición dejada por Manzoni, pues las escritoras apuestan por un tipo de literatura más subversiva desde la que aportan una nueva visión ${ }^{14}$. Tal y como afirma Paola Blelloch (1987: 31), tener en cuenta a estas literatas ${ }^{15}$ es vital para poder comprender la temática que las escritoras contemporáneas reflejan en su literatura: “è necessario risalire alla fine dell'Ottocento quando, dopo un lungo silenzio, esse espressero per la prima volta in letteratura una nuova pressa di coscienza". No hay que olvidar que es en la segunda mitad del Ottocento cuando, en Italia, la questione femminile comienza a cambiar la historia para muchas mujeres, formando pequeños grupos desde los que luchaban para redefinir la sociedad. De hecho, es precisamente durante los años del Risorgimento y finales de este cuando surge un claro movimiento femenino ${ }^{16}$, caracterizado por hacer "cultura e politica con passione: riviste, libelli, conferenze sostengono le battaglie per il voto e l'istruzione femminile" (De Giorgio, 1993: 8). En segundo lugar, y enlazando con la

\footnotetext{
${ }^{14}$ Tal y como Sánchez Dueñas (2008: 17) apunta "Muraro y, a partir de ella Arriaga, estiman que debe priorizarse un concepto de genealogía femenina que incida en nuevas vías creativas que abran cauces para una expresión no imitativa ni hereditaria sino diferenciada, múltiple y sexuada".

${ }^{15}$ Debido a la temática principal del presente estudio, la novela histórica femenina, he omitido escritoras extraordinarias y claves para la lectura de la literatura femenina contemporánea por no practicar dicho género. El estudio de sus obras y contribuciones supondría -y merece- un trabajo aparte, pues evidenciaría una nueva sensibilidad que se despertó a través de la escritura, observando y denunciando la condición de la mujer. No obstante, considero importante citar, al menos, algunas de las grandes protagonistas de finales del Ottocento, verdaderas "madres" de las escritoras contemporáneas como Evelina Cattermole (Contessa Lara), Caterina Percoto, Anna Zuccari Radius (Neera), Maria Antonietta Torriani (Marchesa Colombi) o Emilia Ferretti Viola (Emma). Marina Zancan diferencia claramente dos generaciones de escritoras: en la primera incluye a todas aquellas escritoras que nacidas a mediados del Ottocento, es decir, las arriba mencionadas, y, en la segunda generación entrarían las nacidas por el 1870, como Ada Negri, Grazia Deledda, Sibilla Aleramo, Willy Dias, Anna Franchi, Clarice Tartufari o Annie Vivanti, una lista que la estudiosa integra con las más jóvenes como Maria Messina, Leda Rafanelli, Rosa Rosà, Benedetta. Zancan (2000: 90) afirma que: "Le due generazioni, intrecciate in una fitta rete di relazioni- a tutt'oggi solo in parte ricostruita- tra loro e con le politiche, spesso itineranti tra Milano e Roma, mentre intervengono, come diremo, attivamente nella definizione del modello di donna nuova, nello stesso tempo avviano, parallelamente alla loro sperimentazione letteraria, quella riflessione teorica su soggettività femminile e scrittura che, nel corso del Novecento, si definirà come costante cifra innovativa delle scritture letterarie di donne".

${ }^{16}$ Cfr. Pierini Bortolotti, F., Alle origini del movimento femminile in Italia, 1848-1892, 1963.
} 
última cuestión planteada, las escritoras se alejan de la tradición literaria masculina, y optan por una literatura etiquetada como "ecléctica" por no cumplir con las normas y expectativas de una literatura canonizada bajo un pensamiento masculino, como apunta Marina Zancan (1998) y con la que la crítica literaria no contaba ni se preocupó de buscarle un lugar. Sus narrativas aparecen vinculadas a géneros menores como la novella o el feuilleton ${ }^{17}$, que muchas veces se relaciona más con los sentimientos narrados o la longitud de la obra, dejando la calidad de las obras en un segundo plano.

Por último, y tras haber hecho un breve recorrido por algunas escritoras del Ottocento, muchas de ellas no mencionadas por los motivos expuestos, sí que quisiera matizar que la escritura femenina de este período merece más la pena ser analizada por su contenido femenino y feminista que por cualquier tipo de vinculación que tenga que ver con los géneros literarios. Casalena (2003) ya apuntaba que el Risorgimento consintió a un gran número de italianas, procedentes de diversas formaciones y posiciones socio-económicas, ser visibles $-\mathrm{o}$ al menos, no del todo invisibles- en la sociedad y poder así ser reconocidas como estudiosas y literatas de cierta importancia. La extraordinaria modernidad de pensamiento que mostraron en cada una de sus obras representando la condición femenina a través de los personajes es el gran mérito de estas escritoras. Esa "presa di coscienza" de la que Blelloch habla es precisamente lo que hace de sus obras grandes documentos, no ya sólo desde el punto de vista literario sino también histórico, político y cultural, con unas repercusiones futuras que no dejaran indiferente al público, a la crítica y, cómo no, a escritoras venideras:

\footnotetext{
${ }^{17}$ Respecto a la definición propia de romanzo, Enric Sullà (1996) expone una lúcida reflexión acerca de la controversia que surge entre novella y romanzo. La novella sería un relato de extensión inferior al romanzo, estos criterios puramente cuantitativos se van difuminando en el tiempo. Sin embargo, no todos los estudiosos parecen estar de acuerdo sobre la extensión del romanzo y la novella ya que E.M. Foster (1927) afirma que un romanzo no debe tener menos de cincuenta mil palabras o M. Benedetti (1953) considera que el romanzo debe superar las 150 páginas -45.000 palabras-. Dicha controversia sigue a día de hoy vigente $y$, dado que no es el tema principal que ocupa este estudio, no profundizaré más en el tema. No obstante, y a modo de aclaración, diré que es cierto que las obras asociadas a estas mujeres aparecen preferente catalogadas como novelle, bien por extensión, bien por temática, pero siempre tratando de guardar ciertas distancias con el romanzo. Un futuro estudio sobre el tema debería ser tenido en cuenta pues, si a día de hoy no existe una distinción clara entre novella y romanzo, tal y como afirma Sullà, se debería de prestar más atención a la narrativa histórica escrita por mujeres para (re)descubrir a las "madres de la novela histórica". Es por ello que en este apartado he escogido a las escritoras contemporáneas de Manzoni y que comparten los rasgos de la emergente novela histórica, dando un trato a la historia muy personal y subversivo, más allá de entrar en la polémica del tipo de narración que se escribe.
} 
“[...] le scrittrici degli anni Venti e anche quelle che le precedettero alla fine dell'Ottocento, preparano la strada alla generazione delle scrittrici femministe degli anni Settanta. Esse lasciano loro in eredità dei personaggi femminili spogliati da false sovrastrutture, all'alba di una nuova coscienza, pronti a cambiare la propria condizione e ad affermare la propria individualità" (Blelloch, 1987: 58).

\section{BIBLIOGRAFÍA}

- Arslan, Antonia (1998): Dame, galline e regine. La scrittura femminile italiana tra '800 e '900. Milano: Guerini Studio.

- Arriaga Flórez, Mercedes (2008): “Teorías literarias feministas en Italia”. En: Blas Sánchez Dueñas y María José Porro (coord.): Análisis feministas de la literatura. De las teorías a las prácticas literarias. Córdoba: Universidad de Córdoba, pp. 51-61.

- Bazzoni, Giambattista (1906): Contributo alla storia del romanzo storico italiano. Città di Castello: S. Lapi.

- Bigazzi, Roberto (2007): “Manzoni e Scott”. En: Gianni Oliva (ed.): Manzoni e il realismo europeo. Milano: Mondadori, pp. 36-55.

- Blelloch, Paola (1987): Quel mondo dei guanti e delle stoffe.... Verona: Essedue.

- Casalena, Maria Pia (2003): Scritti storici di donne italiane. Bibliografia 1800-1945. Firenze: Leo S. Olschki Editore.

- Casarini, Giuseppe (2010): “Binasco e il suo Castello: culla del Romanzo storico italiano".

En: Il Corriere della Sera, 24 de marzo, [en línea] Disponible en: http://forum.corriere.it/leggere e scrivere/24-03-2010/binasco-e-il-suo-castelloculla-delromanzo-storico-italiano-1511131.html [15-02-2013].

- Chemello, Adriana (2012): "Luigia Codemo: appunti per una biografia intellettuale". En: Altrelettere, [en línea] Disponible en: http://www.altrelettere.uzh.ch/static/archivio/4 [04-012013].

- Ciopponi, Nadia (2006): Parole di donne. Otto secoli di letteratura italiana al femminile. Milano: Edizioni Clandestine. 
- Costa-Zalesswo, Natalia (1982): Scrittrici italiane dal XIII al XX secolo: testi e critica. Ravenna: Longo Editore.

- Cutrufelli, Maria Rosa (1998): Caracciolo, Enrichetta, Misteri del chiostro napoletano. Nota critica di Maria Rosa Cutrufelli. Firenze: Giunti.

- De Giorgio, Michela (1993): Le italiane dall'Unità a oggi. Roma: Laterza.

- Del Pardo Biezma, Javier (1999): Análisis e Interpretación de La Novela. Madrid: Síntesis

- Eco, Umberto; Federzoni, Marina; Pezzini, Isabella y Pozzato, Maria Pia. (1979) (eds.), Carolina Invernizio, Matilde Serao, Liala. Firenze: La Nuova Italia.

- Fortini, Laura (2010): “Critica femminista e critica letteraria in Italia”. En: Italian Studies, vol. 65,2 , pp.178-191.

- Frye, Northrop (1977): Anatomía de la crítica: cuatro ensayos. Caracas: Monte Ávila editores.

- Ganeri, Margherita (1999): Il romanzo storico in Italia. Il dibattito critico dalle origini al post-moderno. Lecce: Piero Manni.

- Gentile, Giulia (n.d.): "Recensione di Scritti storici di donne italiane. Bibliografia 18001945". En: Donne e conoscenza storica, [en línea] Disponible en: http://www.url.it/donnestoria/testi/recensioni/casgentile.htm [05-02-2013].

- Hutcheon, Linda (1988): A Poetics of Postmodernism: History, Theory, Fiction. London: Routledge.

- Muraro, Luisa (1994): El orden simbólico de la madre. Madrid: horas y HORAS.

- Navarro Salazar, María Teresa (2006): "Mujer e identidad en la narrativa histórica femenina". En: Julio Jurado Morales (ed): Reflexiones sobre la novela histórica. Cádiz: Universidad de Cádiz, pp. 191-219.

- Rivera Garretas, María-Milagros (1996): "El cuerpo indispensable". En: Cuadernos inacabados, $\mathrm{n}^{\circ}$ 24. Madrid: horas y HORAS, pp. 59-71.

- Romano Martín, Yolanda. (2011): “Entre el rosa y el amarillo: la novela folletinesca de Carolina Invernizio". En: Dolores Ramírez Almazán, Milagro Martín Clavijo, Juan Aguilar González y Daniele Cerrato (eds.): La querella de las mujeres en Europa e Hispanoamérica, vol.2. Sevilla: Arcibel, pp. 191-216. 
- Rörig, Karoline (2010): "Gli scritti di Cristina di Belgiojoso tra storiografia e politica”. En: Mariachiara Fugazza y Karoline Rörig (eds.): La prima donna d'Italia. Cristina Trivulzio di Belgiojoso tra politica e giornalismo. Milano: Franco Angeli, pp. 27-60.

- Sánchez Dueñas, Blas (2008): "Bosquejo introductorio. Literatura y feminismos: de las teorías a las prácticas. Fundamentos programáticos y aplicaciones discursivas”. En: Blas Sánchez Dueñas y María José Porro (coord.): Análisis feministas de la literatura. De las teorías a las prácticas literarias. Córdoba: Universidad de Córdoba, pp. 11-32.

- Santoro, Anna (1987): Narratrici italiane dell'Ottocento. Napoli: Federico \& Ardia.

- Smith, Bonnie. G (1996): The Gender of History: Men, Women, and Historical Practice. Cambridge: Harvard University Press.

- Stinghi, Chiara (2001): "La comparsa della donna nella scrittura". En: Letteratatour [en línea] Disponible en: http://www.letteratour.it/tesine/A06donne01.asp [08-01-2013]

- Sullà, Enric (1996) (ed.): Teoría de la novela. Antología de textos del siglo XX. Barcelona: Crítica.

- Trigila, Maria (2004): Letteratura al femminile. Dalle origini ai nostri giorni in Italia. Caltanissetta-Roma: Salvatore Sciascia Editore.

- Wood, Sharon (1995): Italian women's writing 1860-1994. London: Athlone

- Zancan, Marina (2000): "Le autrici. Questioni di scrittura, questioni di letteratura". En: Alberto Asor Rosa (ed.): Letteratura italiana del Novecento. Bilancio di un secolo. Torino: Einaudi, pp. 87-135. 